\title{
Optimal design of robust piezoelectric microgrippers undergoing large displacements
}

\author{
Ruiz, D.; Sigmund, Ole
}

Published in:

Structural and Multidisciplinary Optimization

Link to article, DOI:

$10.1007 / \mathrm{s} 00158-017-1863-5$

Publication date:

2018

Document Version

Peer reviewed version

Link back to DTU Orbit

Citation (APA):

Ruiz, D., \& Sigmund, O. (2018). Optimal design of robust piezoelectric microgrippers undergoing large displacements. Structural and Multidisciplinary Optimization, 75, 71-82. https://doi.org/10.1007/s00158-0171863-5

\section{General rights}

Copyright and moral rights for the publications made accessible in the public portal are retained by the authors and/or other copyright owners and it is a condition of accessing publications that users recognise and abide by the legal requirements associated with these rights.

- Users may download and print one copy of any publication from the public portal for the purpose of private study or research.

- You may not further distribute the material or use it for any profit-making activity or commercial gain

- You may freely distribute the URL identifying the publication in the public portal

If you believe that this document breaches copyright please contact us providing details, and we will remove access to the work immediately and investigate your claim. 
Noname manuscript No.

(will be inserted by the editor)

\section{Optimal design of robust piezoelectric microgrippers undergoing large displacements}

\author{
D. Ruiz • O. Sigmund
}

Received: date / Accepted: date

\begin{abstract}
Topology optimization combined with optimal design of electrodes is used to design piezoelectric microgrippers. Fabrication at micro-scale presents an important challenge: due to non-symmetrical lamination of the structures, out-of-plane bending spoils the behaviour of the grippers. Suppression of this out-ofplane deformation is the main novelty introduced in this work. In addition, a robust approach is used to control length scale in the whole domain and to reduce sensitivity of the design to small fabrication errors. Geometrically non-linear modelling is used for the in-plane deformations whereas out-of-plane motions are modelled by a linear, un-coupled plate model to save computational time. Model and resulting designs are validated by subsequent 3D geometrically non-linear modelling.
\end{abstract}

Keywords Topology optimization · Unimorph · Large displacements · Energy interpolation - Piezoelectric effect $\cdot$ Electrode profile

\section{Introduction}

The conceptual tool of topology optimization has played a very important role in the development of structural design. Nevertheless, its use is not restricted only to this

\section{Ruiz}

E-mail: David.Ruiz@uclm.es

Departamento de Matemáticas, ETSII, Universidad de Castilla-La Mancha, 13071 Ciudad Real, Spain

O. Sigmund

E-mail: sigmund@mek.dtu.dk

Department of Mechanical Engineering, Technical University of Denmark, 2800 Kgs. Lyngby, Denmark field: compliant mechanisms ([Sigmund, 1997],[Frecker et al., 1997],[Jonsmann et al., 1999]), dynamics ([Díaz and Kikuchi, 1992],[Pedersen, 2000]), extremal materials ([Sigmund and Torquato, 1997]) and band gaps ([Sigmund and Jensen, 2003,Jensen and Sigmund, 2011]), amongst others, are fields where its contribution has been crucial.

The topology optimization method has been widely used in the optimal design of MEMS (micro-electromechanical systems), where the size of the devices typically is smaller than 1mm. [Sigmund, 1997] presented optimal design of compliant mechanisms topologies based on the topology optimization method, where optimized mechanisms were fabricated at macro and micro-scale. Concerning piezoelectric effects, [Silva et al., 1997] presented a procedure based on topology optimization and homogenization methods to optimize unit cells for piezocomposites. [Sigmund, 2001a] and [Sigmund, 2001b] optimized thermal and electrothermal microactuators composed of one and two materials, respectively. [Maute and Frangopol, 2003] suggested a methodology for the design of MEMS under stochastic loads and boundary conditions. Recently, [Donoso and Sigmund, 2016] developed a systematic method where a passive gap-phase was included in the optimization process of modal filters for fixed host structure in order to ensure manufacturability and realizability.

The nature of the actuation of the mechanisms in this study is the piezoelectric effect. In the last decades there has been a big development in the subject of topology optimization in piezoelectric materials. The first work where the topology optimization method was used to optimize piezoelectric structures was [Sigmund et al., 1998], that optimized the unit cell of structures for improving piezoelectric features. Regarding piezoelectric actuators, [Silva and Kikuchi, 1999] presented a method to design in-plane actuators by optimizing the host structure, but fixing the piezoelectric material layer. [Kögl and Silva, 2005] presented optimization of piezoelectric layers with a three-layer model, with two piezoelectric films symmetrically bonded to the host structure. [Carbonari et al., 2007] and [Luo et al., 2010] optimized the host structure and the piezoelectric distribution simultaneously. The inclusion of a third variable, the spatial distribution of the control voltage (related to the polarization of the piezoelectric layers) in the optimization problem, was presented in [Kang and Tong, 2008a] and improved in [Kang and Tong, 2008b] by introducing an interpolation scheme in the tri-level actuation voltage term. Further results were presented in [Kang et al., 2011] and [Kang et al., 2012] for in-plane and out-of-plane piezoelectric transducers, respectively. 
In prior works ([Ruiz et al., 2016b], [Ruiz et al., 2016a]) some of the authors have presented a systematic procedure to design static microtransducers and modal filters, respectively. In both works the host structure (including the piezoelectric layer) and polarization profile of the electrodes are optimized simultaneously, considering that both piezoelectric films are perfectly bonded to the top and bottom of the host structure. However, trying to realize this reveals an important obstacle: it is hard to fabricate piezoelectric layers symmetrically in the micro scale ([Kucera et al., 2014]), hence only one piezoelectric film can be deposited on the top of the host structure. This fact is not a problem when the transducer is working as a sensor, since the deformation is produced by an external force. However, when the device is working as actuator it moves in-plane, but also out-of-plane deformation appears, making it challenging to design a genuine microgripper-type actuator. The objective of the present work is to design a piezoelectrically actuated microgripper including constraints on the out-of-plane bending of the structure in some points of interest. In addition, and having manufacturability in mind, the so-called robust formulation ([Sigmund, 2009, Wang et al., 2011]) is applied to the problem with two purposes: first to control the minimum length scale in both solid and void regions; and second to minimize sensitivity to fabrication errors.

Concerning the physical behaviour of the device, we make the assumption that the out-of-plane bending is small but not negligible, meaning that it can be treated by classical linear elasticity plate theory. However, the in-plane displacements are expected to be large and a non-linear model is mandatory. Geometrical non-linearities in topology optimization were first dealt with in [Buhl et al., 2000] using the total Lagrangian formulation. This was soon after extended to compliant mechanism design in [Bruns and Tortorelli, 2001] and [Pedersen et al., 2001]. The robust design of large displacement compliant mechanisms is shown in [Lazarov et al., 2011], where the goal is achieved by adding random variations that model possible geometry errors.

Geometrically non-linear topology optimization is prone to numerical instabilities produced by excessive distortions in low stiffness regions. [Wang et al., 2014] suggested an interpolation scheme that uses linear modelling in the (fictitious) void domain. This scheme is also applied here. In early works ([Buhl et al., 2000] and [Pedersen et al., 2001]) authors avoided the numerical instability by ignoring nodes surrounded by low density elements in the Newton-Raphson convergence criterion. [Bruns and Tortorelli, 2001] circumvented the issue by removing and reintroducing low density elements during the optimization procedure. Removal of low density elements may come at the risk of hindering structure to grow in low density regions and hence may be better suited for more shape oriented topology optimization approaches like [Zhang et al., 2017].

The paper is organized as follows. In Section 2 the nature of the problem is briefly described and the discrete formulation is shown. Section 3 is devoted to the robust formulation. In Section 4 we present the numerical implementation of the problem, where the algorithm used to get the optimal designs is described. The optimized microgrippers obtained by solving the discrete problem are shown and validated in Comsol Multiphysics in Section 5. Finally, in Section 6 the conclusions of this work are presented.

\section{Topology optimization of large displacement piezoelectric microgripper}

As design domain $\Omega$ we consider a rectangular plate clamped at its left side. On the top surface, the host structure is perfectly bonded with a piezoelectric layer (that is sandwiched between two electrodes) of negligible stiffness compared to the plate.

The polarization of the piezoelectric layer is obtained through the application of an input voltage over the electrodes. This voltage generates an electric field that produces a mechanical stress over the piezoelectric material, which in turn deforms the host structure. The unsymmetrical configuration of the layers of the plate causes bending of the structure, that disturbs the in-plane behaviour and needs to be suppressed. The multilayered structure is shown in Fig.1. Three passive areas are defined in the design domain. Two of these areas are solid regions (black colour) that belong to the jaws. The third one is a void region (white colour) and represents an empty gap between the jaws. The output of the gripper is modelled by a spring of stiffness $k_{\text {out }}$ (that depends on the application). The $3 \mathrm{~d}$ depiction of design domain and the dimensions are shown in Fig.2.

The aim of the problem is the maximization of the in-plane deformation along the $y$-axis $u_{1}$ while the deformations over the $z$-axis $u_{2}$ and $u_{3}$ must be suppressed. The bending is cancelled in two points in order to avoid the rotation of the jaws over the $y$-axis. This suppression is controlled by adding two constraints which relate the optimized and the cancelled displacements. In addition, a volume constraint is used to control the amount of material used.

The optimization problem involves two design variable fields. $\chi_{s}$ is a characteristic function, $\chi_{s} \in\{0,1\}$, that represents the structure layout $\left(\chi_{s}=1\right)$ and void $\left(\chi_{s}=0\right)$, as usual. $\chi_{p}$ is also a characteristic function 


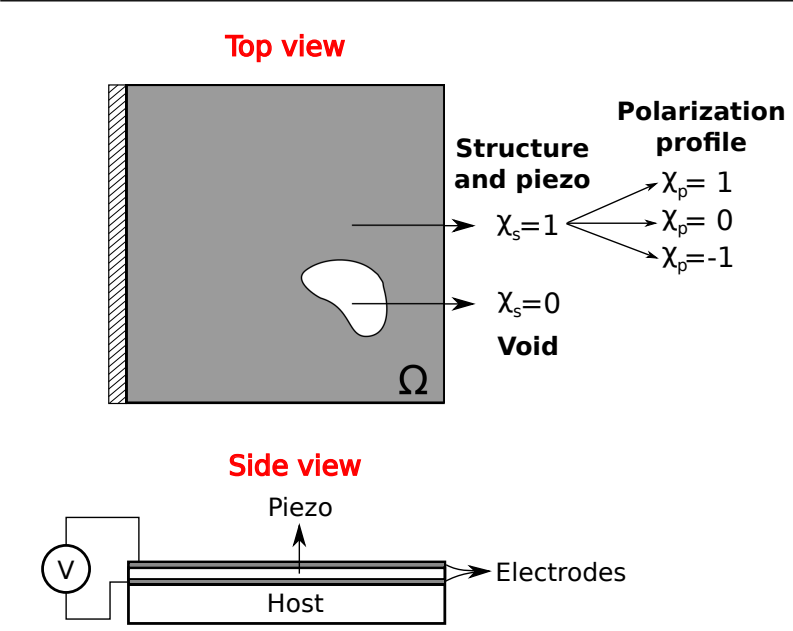

Fig. 1 Top and side view of the piezoelectric device.

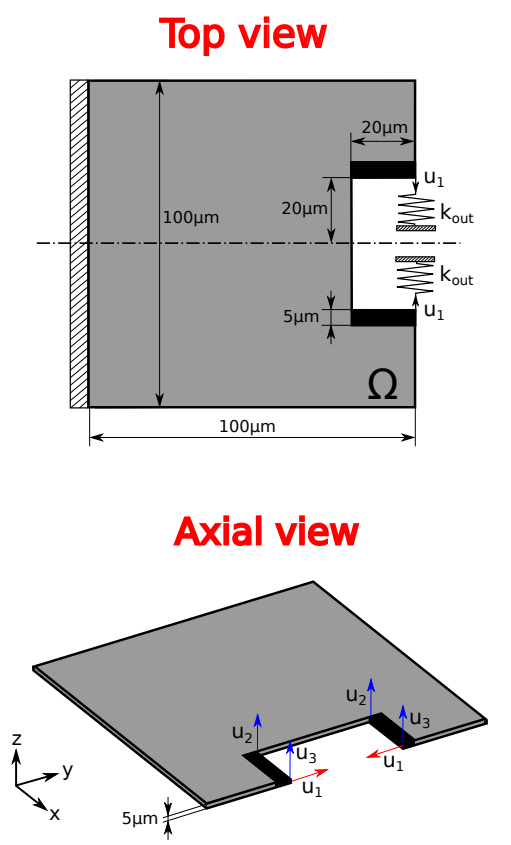

Fig. 2 Design domain.

such that $\chi_{p} \in\{-1,0,1\}$, meaning negative, null or positive polarity. Since the topology optimization problem lacks classical solutions, the characteristic functions $\chi_{s}$ and $\chi_{p}$ need to be relaxed into density variables $\rho_{s} \in[0,1]$ and $\rho_{p} \in[-1,1]$. As usual, after the relaxation of the variables, the domain is discretized in $n_{e}$ finite elements with two variables per element. The role of the electrode is crucial here, only the parts of the structure that are being covered by electrode, i.e. $\chi_{p}=-1$ or $\chi_{p}=1$, are electrically affected and therefore subjected to piezoelectric forces.

Since the out-of-plane deformation is expected to be small, it can be studied separately from the in-plane one (the problems are decoupled), and then the optimization problem involves two equilibrium equations. The in-plane displacements are modelled using a geometrically non-linear model. The out-of-plane displacements are modelled using classical linear elasticity. The formulation of the problem written as a topology optimization problem becomes:

$\max _{\boldsymbol{\rho}_{s}, \boldsymbol{\rho}_{p}}: u_{1}\left(\boldsymbol{\rho}_{s}, \boldsymbol{\rho}_{p}\right)$

$$
\begin{aligned}
\text { s.t. : } \mathbf{R}_{i p}\left(\boldsymbol{\rho}_{s}, \boldsymbol{\rho}_{p}, \mathbf{U}_{i p}\right) & =\mathbf{0} \\
\mathbf{R}_{o p}\left(\boldsymbol{\rho}_{s}, \boldsymbol{\rho}_{p}, \mathbf{U}_{o p}\right) & =\mathbf{0} \\
u_{1} & =\mathbf{L}_{1}^{T} \mathbf{U}_{i p} \\
u_{2} & =\mathbf{L}_{2}^{T} \mathbf{U}_{o p} \\
u_{3} & =\mathbf{L}_{3}^{T} \mathbf{U}_{o p} \\
\left(\frac{u_{2}}{u_{1}}\right)^{2}-\varepsilon_{d}^{2} & \leq 0 \\
\left(\frac{u_{3}}{u_{1}}\right)^{2}-\varepsilon_{d}^{2} & \leq 0 \\
\frac{\mathbf{1}^{T} \boldsymbol{\rho}_{s}}{V}-1 & \leq 0 \\
\boldsymbol{\rho}_{s} & \in[\mathbf{0}, \mathbf{1}] \\
\boldsymbol{\rho}_{p} & \in[-\mathbf{1}, \mathbf{1}],
\end{aligned}
$$

where $\boldsymbol{\rho}_{s}$ and $\boldsymbol{\rho}_{p}$ represent the structure layout and the polarization profile respectively, $u_{1}$ is the in-plane displacement to be maximized, $u_{2}$ and $u_{3}$ are the outof-plane displacements to be suppressed, $\mathbf{L}_{1}, \mathbf{L}_{2}$ and $\mathbf{L}_{3}$ are vectors of zeros with 1 in the output degree of freedom of interest, $V$ is the maximum volume, $\varepsilon_{d}$ is a small value that relates the displacements to be suppressed and the ones to be optimized, and $\mathbf{R}_{i p}$ and $\mathbf{R}_{o p}$ are the global residual vectors of the structural equilibrium equations for the in-plane and out-of-plane cases, respectively. For the sake of brevity, from now on the subscripts "ip" and "op" refer to the in-plane and the out-of-plane cases, respectively. The residual vectors are defined as follows:

$$
\begin{aligned}
\mathbf{R}_{i p} & =\mathbf{F}_{i p}^{p i e z o}-\mathbf{F}_{i p}^{i n t}=\mathbf{0} \\
\mathbf{R}_{o p} & =\mathbf{F}_{o p}^{p i e z o}-\mathbf{F}_{o p}^{i n t}=\mathbf{0}
\end{aligned}
$$

where $\left(\mathbf{F}_{i p}^{\text {piezo }}, \mathbf{F}_{o p}^{\text {piezo }}\right)$ and $\left(\mathbf{F}_{i p}^{i n t}, \mathbf{F}_{o p}^{i n t}\right)$ are the global piezoelectric and internal force vectors, respectively. Capital letters are used for global vectors and matrices, that are obtained by assembling the elemental contributions represented with lowercase letters. The expressions of the different terms in equation (2) are further elaborated in the next subsection. 
2.1 Continuous material approach and finite element model

The original formulation of the problem involves integer variables which need to be relaxed into density variables. The well-known SIMP approach (Solid Isotropic Material with Penalization, [Bendsøe and Sigmund, 1999] and [Bendsøe and Sigmund, 2003]) is used for this purpose. The Young's modulus of each element depends on the element density as follows:

$E_{e}=\left(\bar{\rho}_{s e}\right)^{p}\left(E_{0}-E_{m i n}\right)+E_{m i n}$,

where $E_{0}$ is the Young's modulus of the base material, $E_{\text {min }}>0$ is a small value used to avoid singularities in the stiffness matrix and $p$ is the penalization exponent (typically $p=3$ ).

The discrete formulation of the problem has been presented in Section 2. For the in-plane case bilinear elements with 8 degrees of freedom are used and for the out-of-plane case, Kirchhoff elements with 12 degrees of freedom per element are used. The in-plane displacements are expected to be large in comparison with the size of the domain. [Pedersen et al., 2001] showed that displacements over $2 \%$ of the domain size require geometrical non-linearity to model the deformation field.

In order to model the in-plane deformation, the total Lagrangian finite element formulation is used. The Green-Lagrange strain tensor can be expressed as:

$\mathbf{E}=\frac{1}{2}\left(\mathbf{F F}^{T}-\mathbf{I}\right)$,

where $\mathbf{I}$ is the unit tensor and $\mathbf{F}$ is the deformation gradient, defined by a $2 \times 2$ matrix:

$\mathbf{F}=\partial u / \partial y=\left[\begin{array}{l}\frac{\partial u}{\partial x} \frac{\partial u}{\partial y} \\ \frac{\partial v}{\partial x} \frac{\partial v}{\partial y}\end{array}\right]$

The expression for the work conjugate stress tensor (the second Piola-Kirchhoff stress tensor) is:

$\mathbf{S}=\frac{\partial \phi\left(\mathbf{U}_{i p}\right)}{\partial \mathbf{E}}$,

where $\phi\left(\mathbf{U}_{i p}\right)$ is the in-plane stored elastic energy density that will be defined in the next subsection.

The expression for the element internal force $\mathbf{f}_{i p}^{\text {int }}$ presented in equation (2) is:

$\mathbf{f}_{i p}^{i n t}=\frac{\partial \int_{\Omega} \phi_{e} \mathrm{~d} \Omega}{\partial \mathbf{u}_{i p}}$,

where $\mathbf{u}_{i p}$ is the element displacement vector for the in-plane case.
Due to the non-symmetrical laminate, the piezoelectric force generates a moment that makes the plate bend and an initial strain that extends or contracts the plate. All expressions for the computations of both the flexural and the extensional components, can be found in [Gibbs and Fuller, 1992]. In addition, the same powerlaw dependence $R_{e}=\rho_{s e}^{p}$ as used for the Young's modulus interpolation equation (3) is used for the piezoelectric force generation. It is easy to see that the value of the interpolation $R_{e}$ is very small in void regions and takes the value $R_{e}=1$ in the solid ones. This is a realistic way to model the force produced in these elements, since there is no electrode in the void regions [Ruiz et al., 2016b].

In the equilibrium configuration, the residual vector for each case must be 0 , this means that the global vectors of internal forces must be equal to the piezoelectric forces. We can rewrite the equilibrium equation for each motion case as follows:

$$
\begin{aligned}
& \mathbf{R}_{i p}=\mathbf{F}_{i p}^{\text {piezo }}-\mathbf{F}_{i p}^{i n t}=\mathbf{F}_{i p}^{\text {piezo }}-\int_{\Omega} \mathbf{B}_{i p}\left(\mathbf{U}_{i p}\right) \mathbf{S ~ d} \Omega \\
& \mathbf{R}_{o p}=\mathbf{F}_{o p}^{\text {piezo }}-\mathbf{F}_{o p}^{i n t}=\mathbf{F}_{o p}^{\text {piezo }}-\mathbf{K}_{o p} \mathbf{U}_{o p},
\end{aligned}
$$

where $\mathbf{K}_{o p}$ is the global out-of-plane stiffness matrix obtained by assembling the element stiffness matrices and $\mathbf{B}_{i p}$ is the in-plane non-linear strain displacement matrix. The Newton-Raphson method is used to solve the non-linear system:

$\mathbf{K}_{t} \Delta \mathbf{U}_{i p}=\mathbf{R}_{i p}$

where $\mathbf{K}_{t}$ is the tangent stiffness matrix defined as:

$\mathbf{K}_{t}=-\frac{\partial \mathbf{R}_{i p}}{\partial \mathbf{U}_{i p}}$

and the nodal displacement vector is updated by $\mathbf{U}_{i p}=$ $\mathbf{U}_{i p}+\Delta \mathbf{U}_{i p}$. The detailed computations of the tangent matrix and the nodal force vectors can be found on [Zienkiewicz et al., 2014] and are not stated here.

\subsection{Energy interpolation scheme}

The Saint-Venant-Kichhoff model is used to represent the behaviour of the hyperelastic material. The stored elastic energy density is expressed as follows:

$\phi=\frac{1}{2} \lambda E_{k k}^{2}+\mu E_{i j} E_{i j}=$

$\frac{1}{8} \lambda\left(I_{c}-3\right)^{2}+\frac{\mu}{4}\left(I_{c}^{2}-2 I I_{c}-2 I_{c}+3\right)$,

where $E_{i j}$ are the components of the non-linear strain tensor, $\lambda$ and $\mu$ are the Lamé parameter, $I_{c}=\operatorname{tr}(\mathbf{C})$ is the first invariant of $\mathbf{C}=\mathbf{F}^{T} \mathbf{F}$ and $I I_{c}=\left(\operatorname{tr}(\mathbf{C})^{2}-\right.$ 
$\left.\operatorname{tr}\left(\mathbf{C}^{2}\right)\right) / 2$ is the second invariant of $\mathbf{C}$. The Lamé parameters can be expressed in function of the Young's modulus and the Poisson's ratio: $\lambda=\nu E\left(1-\nu^{2}\right)$ and $\nu=E /(2(1+\nu))$.

In [Wang et al., 2014] an energy interpolation scheme is used to alleviate the issue of distorted and ill-converged void region mesh. The paper suggests basing the analysis in the solid region on the non-linear stored energy and the analysis in the void regions on linear stored energy, thereby eliminating mesh distortion and ill-convergence issues in the low density domain. The energy interpolation form for element $e$ is

$\Phi_{e}\left(\mathbf{u}_{e}\right)=\left[\Phi\left(\gamma_{e} \mathbf{u}_{e}\right)-\Phi_{L}\left(\gamma_{e} \mathbf{u}_{e}\right)+\Phi_{L}\left(\mathbf{u}_{e}\right)\right] E_{e}$,

where $E_{e}$ is the Young's modulus for element $e, \mathbf{u}_{e}$ is the elemental displacement vector for the element, $\Phi(\cdot)$ is the stored elastic energy density, $\Phi_{L}(\cdot)$ is the stored elastic energy density under small deformation, both with unit Young's modulus. Finally, $\gamma_{e}$ is the interpolation factor that takes the value $\gamma_{e}=1$ if the element is solid and $\gamma_{e}=0$ if it is void. This interpolation scheme assumes that the Young's modulus is separable from the energy functional. In order to differ between void and solid regions a smoothed Heaviside projection is used:

$\gamma_{e}=\frac{\tanh \left(\beta_{1} \rho_{0}\right)+\tanh \left(\beta_{1}\left(\bar{\rho}_{s e}^{p}-\rho_{0}\right)\right)}{\tanh \left(\beta_{1} \rho_{0}\right)+\tanh \left(\beta_{1}\left(1-\rho_{0}\right)\right)}$,

where $\bar{\rho}_{s e}$ is the physical density, which will be introduced in the next section, $\beta_{1}$ models the smoothness of the projection and $\rho_{0}$ is a low-density threshold. For non-void domains $\bar{\rho}_{s e}^{p}>\rho_{0}$ the element is hence modelled as a standard non-linear element with geometrically non-linear contribution.

\section{Robust topology optimization formulation}

This section is devoted to the robust formulation of the problem. This approach, that was presented in [Sigmund, 2009, Wang et al., 2011], consists in the use of three different projections with two goals. The first one is controlling the minimum length scale in both, solid and void regions hence avoiding the appearance of hinges. The second is the robustness towards small manufacturing errors, that are very common in the fabrication at micro-scale.

The robust approach proposes the use of three different projections: eroded, intermediate and dilated. The expression for a smoothed threshold projection based on the tanh function is

$\bar{\rho}_{s e}=\frac{\tanh \left(\beta_{0} \eta\right)+\tanh \left(\beta_{0}\left(\tilde{\rho}_{s e}-\eta\right)\right)}{\tanh \left(\beta_{0} \eta\right)+\tanh \left(\beta_{0}(1-\eta)\right)}$, where $\beta_{0}$ is a tuning parameter representing the smoothness of the projection and $\eta$ is the threshold which can take values between 0 and 1 . The filtered densities $\tilde{\rho}_{s e}$ are projected to 1 if these values are bigger than the threshold and to 0 if not. The filtered densities $\tilde{\rho}$ are expressed as:

$\tilde{\rho}_{s e}=\frac{\sum_{j \in N_{e}} w\left(\mathbf{x}_{j}\right) v_{j} \rho_{s j}}{\sum_{j \in N_{e}} w\left(\mathbf{x}_{j}\right) v_{j}}$,

where $\mathbf{x}_{j}$ is the center of element $j, v_{j}$ is the volume of the element $j, N_{e}$ is the neighbourhood of element $e$ within a certain filter radius $r$ specified by $N_{e}=$ $\left\{j \mid\left\|\mathbf{x}_{j}-\mathbf{x}_{e}\right\| \leq r\right\}$, and $w\left(\mathbf{x}_{j}\right)=r-\left\|\mathbf{x}_{j}-\mathbf{x}_{e}\right\|$.

The use of three different physical realizations requires the solution of three sets of equilibrium equations. Followingly, each realization presents three different in-plane and out-of-plane displacements which all must be included in the optimization problem. From now on, for the sake of simplicity, we introduce the superscript $q$ for indicating projection, with $e$ meaning erode, $i$ intermediate and $d$ dilate. The robust topology optimization formulation is written:

$\max _{\boldsymbol{\rho}_{s}, \boldsymbol{\rho}_{p}}: \min _{q=e, i, d}\left\{u_{1}^{q}\left(\overline{\boldsymbol{\rho}}_{s}^{q}, \boldsymbol{\rho}_{p}\right)\right\}$

s.t.:

$$
\begin{aligned}
\mathbf{R}_{i p}^{q}\left(\overline{\boldsymbol{\rho}}_{s}^{q}, \boldsymbol{\rho}_{p}, \boldsymbol{U}_{i p}^{q}\right) & =\mathbf{0} \\
u_{1}^{q} & =\mathbf{L}_{1}^{T} \mathbf{U}_{i p}^{q} \\
\mathbf{R}_{o p}^{q}\left(\overline{\boldsymbol{\rho}}_{s}^{q}, \boldsymbol{\rho}_{p}, \boldsymbol{U}_{o p}^{q}\right) & =\mathbf{0} \\
u_{2}^{q} & =\mathbf{L}_{2}^{T} \mathbf{U}_{o p}^{q} \\
u_{3}^{q} & =\mathbf{L}_{3}^{T} \mathbf{U}_{o p}^{q} \\
\left(\frac{u_{2}^{q}}{u_{1}^{q}}\right)^{2}-\varepsilon_{d}^{2} & \leq 0 \\
\left(\frac{u_{3}^{q}}{u_{1}^{q}}\right)^{2}-\varepsilon_{d}^{2} & \leq 0 \\
\frac{\mathbf{1}^{T} \overline{\boldsymbol{\rho}}_{s}^{d}}{V_{d}^{*}-1} & \leq 0 \\
\boldsymbol{\rho}_{s} & \in[\mathbf{0}, \mathbf{1}] \\
\boldsymbol{\rho}_{p} & \in[-\mathbf{1}, \mathbf{1}] \\
q & \equiv e, i, d ;
\end{aligned}
$$

where $V_{d}^{*}$ is the maximum volume bound over the dilated design. This value is computed at the beginning of the optimization and is then updated every 20 iterations. The expression for this constraint is

$V_{d}^{*}=\frac{V^{*}}{V_{i}} V_{d}$

with $V_{i}$ and $V_{d}$ being the volume for the intermediate and dilated designs and $V^{*}$ the maximum volume 
prescribed for the intermediate design. Unlike the equilibrium equations and the rest of the constraints, the volume constraint is only enforced on the dilated design, according to the method proposed by [Wang et al., 2011].

The max-min objective function proposed above is not differentiable. In order to alleviate this issue the problem is reformulated using the so-called bound formulation:

$\min _{\boldsymbol{\rho}_{s}, \boldsymbol{\rho}_{p}}: \beta$

s.t.:

$$
\begin{aligned}
-u_{1}^{q} & \leq \beta \\
\mathbf{R}_{i p}^{q}\left(\overline{\boldsymbol{\rho}}_{s}^{q}, \boldsymbol{\rho}_{p}, \boldsymbol{U}_{i p}^{q}\right) & =0 \\
u_{1}^{q} & =\mathbf{L}_{1}^{T} \mathbf{U}_{i p}^{q} \\
\mathbf{R}_{o p}^{q}\left(\overline{\boldsymbol{\rho}}_{s}^{q}, \boldsymbol{\rho}_{p}, \boldsymbol{U}_{o p}^{q}\right) & =0 \\
u_{2}^{q} & =\mathbf{L}_{2}^{T} \mathbf{U}_{o p}^{q} \\
u_{3}^{q} & =\mathbf{L}_{3}^{T} \mathbf{U}_{o p}^{q} \\
\left(\frac{u_{2}^{q}}{u_{1}^{q}}\right)^{2}-\varepsilon_{d}^{2} & \leq 0 \\
\left(\frac{u_{3}^{q}}{u_{1}^{q}}\right)^{2}-\varepsilon_{d}^{2} & \leq 0 \\
\frac{\mathbf{1}^{T} \overline{\boldsymbol{\rho}}_{s}^{d}}{V_{d}^{*}-1} & \leq 0 \\
\boldsymbol{\rho}_{s} & \in[\mathbf{0}, \mathbf{1}] \\
\boldsymbol{\rho}_{p} & \in[-\mathbf{1}, \mathbf{1}] \\
q & \equiv e, i, d ;
\end{aligned}
$$

where $\beta$ is an additional bound variable that does not depend on the design variables $\boldsymbol{\rho}_{s}$ and $\boldsymbol{\rho}_{p}$ and resolves non-differentiability issue with the max-min function.

\section{Numerical implementation}

A gradient-based method, the MMA (Method of Moving Asymptotes [Svanberg, 1987]), has been used to solve the optimization problem. This method requires information about the objective function, the constraints and the sensitivities of both. The adjoint method is used to compute the sensitivities of the objective function with respect to the structure density vector $\boldsymbol{\rho}_{s}$ and the polarization profile vector $\boldsymbol{\rho}_{p}$. First the derivative with respect to the structural density of element $e$ are found using adjoint sensitivity analysis as

$\frac{\partial u_{1}^{q}}{\partial \rho_{s e}}=\lambda^{T} \frac{\partial \mathbf{R}_{i p}^{q}}{\partial \rho_{s e}}$

with

$\mathbf{K}_{t} \boldsymbol{\lambda}=\left(\frac{\partial u_{1}^{q}\left(\mathbf{U}_{i p}^{q}, \boldsymbol{\rho}_{s}, \boldsymbol{\rho}_{p}\right)}{\partial \mathbf{U}_{i p}^{q}}\right)=\mathbf{L}_{1}$, where the tangent matrix $\mathbf{K}_{t}$ is computed at the converged solution. The right term in equation (4) is the constant vector $\mathbf{L}_{1}$. The chain rule must be used to compute the derivatives of the equilibrium equation:

$$
\begin{aligned}
& \frac{\partial \mathbf{R}_{i p}^{q}}{\partial \rho_{s e}}=\frac{\partial \mathbf{r}_{i p}^{q}}{\partial \rho_{s e}}=-\sum_{j \in N_{e}}\left(\frac{\partial\left(\mathbf{f}_{i p, j}^{i n t, q}-\mathbf{f}_{i p, j}^{p i e z o, q}\right)}{\partial \bar{\rho}_{s j}^{q}}\right. \\
& \left.+\frac{\partial\left(\mathbf{f}_{i p, j}^{i n t, q}-\mathbf{f}_{i p, j}^{p i e z o, q}\right)}{\partial \gamma_{j}^{q}} \frac{\partial \gamma_{j}^{q}}{\partial \bar{\rho}_{s j}^{q}}\right) \frac{\partial \bar{\rho}_{s j}^{q}}{\partial \tilde{\rho}_{s j}} \frac{\partial \tilde{\rho}_{s j}}{\partial \rho_{s e}} .
\end{aligned}
$$

Due to the many load cases and realizations involved, indexing above has become quite involved. Information about the nature of the force (internal or piezoelectric) and the threshold (eroded, intermediate or dilated) is indicated as superscripts, separated by a comma. Information about the motion case (in-plane or out-of-plane) and the element number $(j)$ is shown in the subscript, also separated by a comma.

In the same way, the derivative of the cost with respect to the polarization profile is:

$$
\frac{\partial u_{1}^{q}}{\partial \rho_{p e}}=\lambda^{T} \frac{\partial \mathbf{R}_{i p}^{q}}{\partial \rho_{p e}}
$$

with the adjoint vector $\boldsymbol{\lambda}$ again coming from the solution of (4).

Finally, the derivative of the residual vector is

$$
\frac{\partial \mathbf{R}_{i p}^{q}}{\partial \rho_{p e}}=\frac{\partial \mathbf{r}_{i p}^{q}}{\partial \rho_{p e}}=-\frac{\partial\left(\mathbf{f}_{i p}^{i n t, q}-\mathbf{f}_{i p}^{\text {piezo,q}}\right)}{\partial \rho_{p e}}
$$

The sensitivities of $u_{2}^{q}$ and $u_{3}^{q}$ are also computed using the adjoint method. However, since the out-of-plane problem is linear, these computations are straight forward and are not stated here. Similarly, the volume fraction depends linearly on the physical structure density, and the derivatives are computed using the chain rule.

The complete process algorithm looks like:

1. Selection of the dimensions of the plate and the properties of the materials that will be used. Boundary conditions and the parameters $\varepsilon_{d}$ and $k_{\text {out }}$ must be chosen.

2. Initialize the design variables $\boldsymbol{\rho}_{s}$ and $\boldsymbol{\rho}_{p}$.

3. Compute the physical densities $\overline{\boldsymbol{\rho}}_{s}^{q}$ by filtering the structural density and then projecting with three different thresholds.

4. Solve the finite element problem for the three different physical densities.

(a) For the linear out-of-plane case.

(b) For the non-linear in-plane case.

5. Extract the displacements $u_{1}^{q}, u_{2}^{q}$ and $u_{3}^{q}$ and compute the constraints. 
6. Compute the sensitivities of the objective function and the constraints.

7. Update design variables based on MMA.

8. Until convergence update the parameters $\beta_{0}$ and $V_{d}^{*}$ and go back to step 3 .

At this point it is important to remark that thanks to the symmetry of the problem only half of the domain needs to be simulated and optimized.

\section{Examples}

The multilayered structure, whose dimensions are shown in Fig.1, is formed in a host layer of silicon with thickness $t=5 \mu \mathrm{m}$ and a piezoelectric layer of PZT-5H with thickness $t_{p}=500 \mathrm{~nm}$. The Young's modulus is $E_{0}=$ $130 \mathrm{GPa}$ for silicon, the stiffness of the piezoelectric layer is neglected. In order to avoid singularities in the stiffness matrix, we fix $E_{\min }=10^{-9} E_{0}$. The Poisson's ratio is $\nu=0.28$ for both materials. The piezoelectric constant for PZT-5A is $d_{31}=190 \mathrm{pm} / \mathrm{V}$ and the input voltage is $V_{i n}=500 \mathrm{~V}$. We fix the relationship between the suppressed and optimized displacement to $\varepsilon_{d}=0.05$. The stiffness of the spring that models the output is $k_{\text {out }}=1 \times 10^{3} \mathrm{~N} / \mathrm{m}$. Concerning the filter and the projection, the radius filter is set to $r=8 \mu \mathrm{m}$ and $\delta \eta=0.30$, ensuring a minimum length scale of $7.2 \mu \mathrm{m}$ ([Wang et al., 2011] and [Qian and Sigmund, 2013]) for the intermediate design. Threshold projection values of $\eta=0.7,0.5$ and 0.3 are hence assigned to the eroded $\overline{\boldsymbol{\rho}}_{s}^{e}$, intermediate $\overline{\boldsymbol{\rho}}_{s}^{i}$ and dilated $\overline{\boldsymbol{\rho}}_{s}^{d}$ designs, respectively. The value of the sharpness parameter $\beta_{0}$ is increased during the iterative process, starting with $\beta_{0}=1$ and doubling each 20 iterations until it reaches $\beta_{0}=16$. Finally, we fix the parameters of the energy interpolation scheme to $\beta_{1}=500$ and $\rho_{0}=0.01$ ([Wang et al., 2014]).

The optimized designs for the first example are given in Fig. 3. Three different thresholds are shown: the intermediate (top), also called the blueprint design which is the one that will be fabricated, the eroded (middle) and the dilated (bottom). The structural layout $\overline{\boldsymbol{\rho}}_{s}$ is shown in Fig. 3 (left), where black and white means solid and void areas, respectively. There is no microstructure (gray), which means that the projection method is working properly. Fig. 3 (right) shows the electrode profile $\boldsymbol{\rho}_{p}$. Orange and cyan represent the sign of the polarization profile, positive or negative respectively. The whole structure, except for the jaws, is being covered by electrode. The value of the in-plane displacement in the blueprint design is $u_{1}=13.9 \mu \mathrm{m}$. The values of the out-of-plane displacements are $u_{2}=0.44 \mu \mathrm{m}$ and $u_{3}=0.69 \mu \mathrm{m}$.

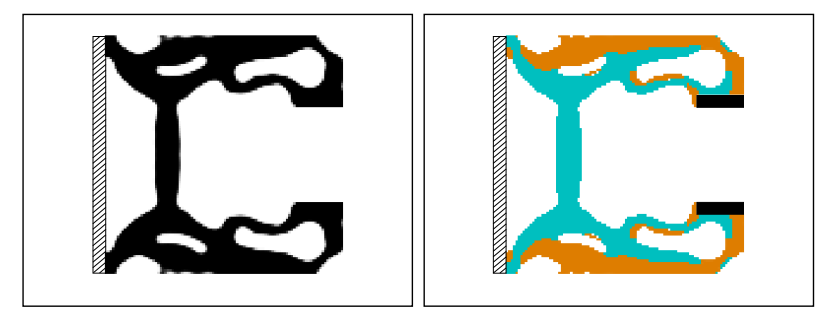

(a) Intermediate projection.

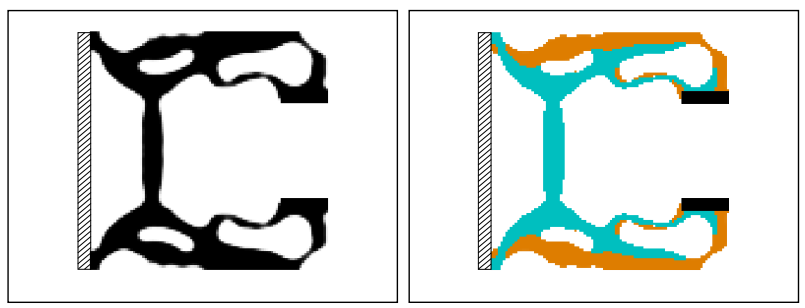

(b) Erode projection.

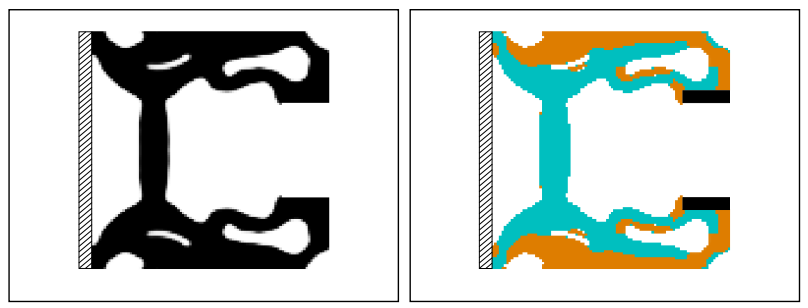

(c) Dilate projection.

Fig. 3 Structure variable $\overline{\boldsymbol{\rho}}_{s}$ (left) and electrode profile $\boldsymbol{\rho}_{p}$ (right) for the three different projections. $k_{\text {out }}=1 \times 10^{3} \mathrm{~N} / \mathrm{m}$.

In Fig.4 the stiffness of the spring is changed to $k_{\text {out }}=5 \times 10^{2} \mathrm{~N} / \mathrm{m}$ while the rest of the parameters stay fixed. The value of the displacement in the blueprint design is $u_{1}=18 \mu \mathrm{m}$.

Finally, in Fig.5, the stiffness of the spring is set to $k_{\text {out }}=5 \times 10^{3} \mathrm{~N} / \mathrm{m}$ and the value of the optimized displacement is $u_{1}=6 \mu \mathrm{m}$.

In Fig. 6 and Fig. 7 the response of the three optimized grippers depending on the output stiffness $k_{\text {out }}$ is shown. In both figures the in-plane equilibrium equation is solved for different values of the stiffness of the spring. In the first case the values of $u_{1}$ versus $k_{\text {out }}$ are plotted for the blueprint design. When the stiffness takes the value $k_{\text {out }}=0 \mathrm{~N} / \mathrm{m}$, the displacement obtained is the so-called free displacement. As expected, the device designed for the lowest stiffness (red colour) shows the biggest free displacement, and vice versa. In the second case, the values of force applied at the end of the jaws versus $k_{\text {out }}$ are plotted. When the stiffness takes a big value, the displacement at this point tends to zero and the resulting force is called blocking force. The device designed for the biggest stiffness (blue colour) presents the biggest blocking force. These two parameters, free displacement and blocking force, de- 


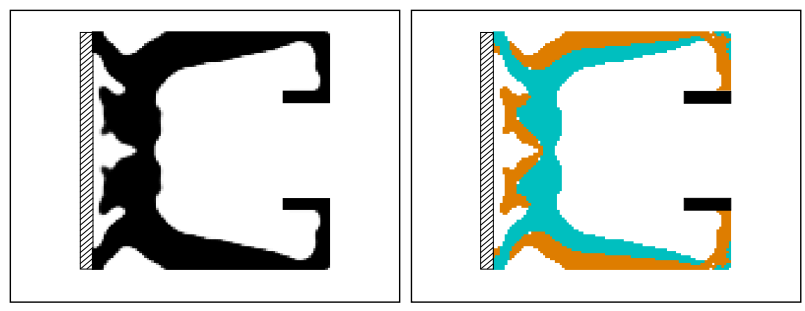

(a) Intermediate projection.

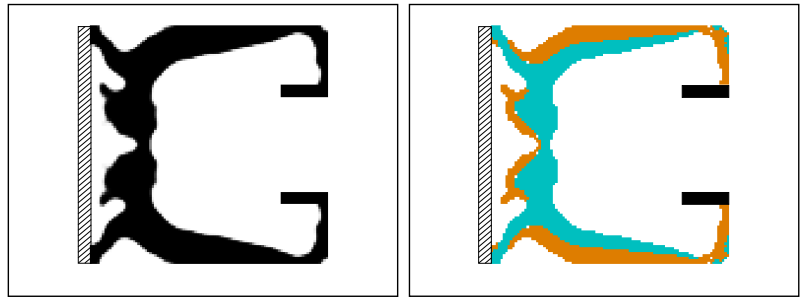

(b) Erode projection.

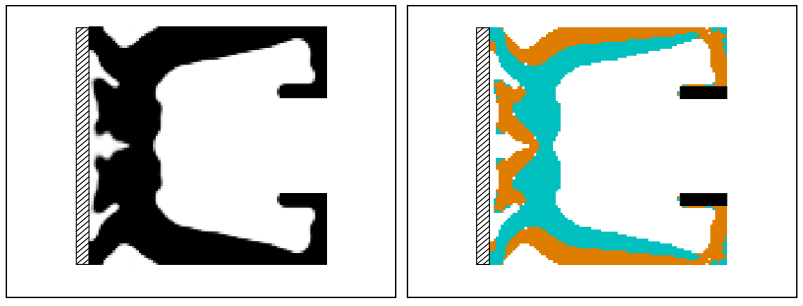

(c) Dilate projection.

Fig. 4 Structure variable $\overline{\boldsymbol{\rho}}_{s}$ (left) and electrode profile $\boldsymbol{\rho}_{p}$ (right) for the three different projections. $k_{\text {out }}=5 \times 10^{2} \mathrm{~N} / \mathrm{m}$.

fine the behaviour of the gripper. Notice that the best design (the highest displacement in the first case, and the force applied in the second one) for the three different stiffness's, is the one corresponding to the designed spring.

In order to further illustrate the potential of our approach, Fig. 8 presents two other optimized microgrippers. Fig. 8 (left) shows the one optimized for $k_{\text {out }}=$ $1 \times 10^{3} \mathrm{~N} / \mathrm{m}$ and Fig. 8 (right) shows the same example but removing the constraints over the out-of-plane displacements. The topologies are quite similar, actually the in-plane displacement $u_{1}$ is $13.9 \mu \mathrm{m}$ for the first case and $15 \mu \mathrm{m}$ for the second one, but the out-of-plane displacements $u_{2}$ and $u_{3}$ are very different. When these displacements are constrained their values in the optimized design are $u_{2}=0.44 \mu \mathrm{m}$ and $u_{3}=0.69 \mu \mathrm{m}$. When both constraints are removed from the optimization problem, the two displacements change to $u_{2}=8.4 \mu \mathrm{m}$ and $u_{3}=12.8$. In such a case, and taking into account the size of the plate, the in-plane and out-of-plane problems are not decoupled. In this simulation the model used is not the most appropriate, but anyway, the objective of the present work is showing that the opti-

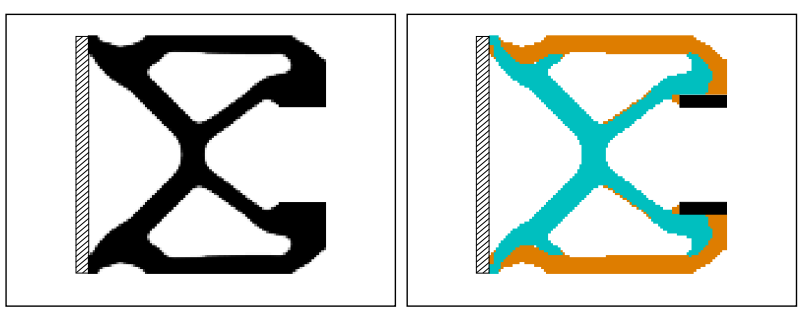

(a) Intermediate projection.

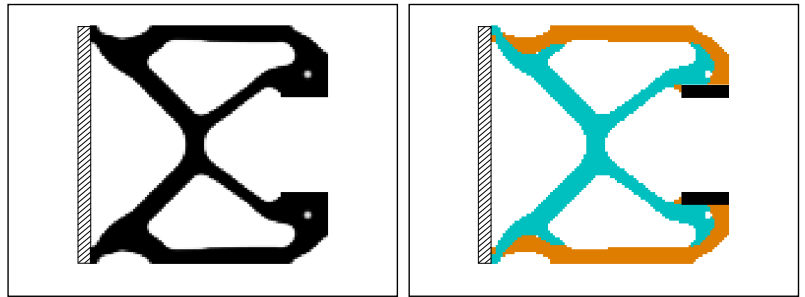

(b) Erode projection.

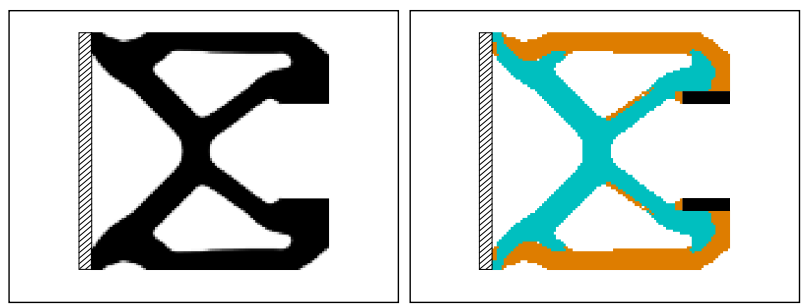

(c) Dilate projection.

Fig. 5 Structure variable $\overline{\boldsymbol{\rho}}_{s}$ (left) and electrode profile $\boldsymbol{\rho}_{p}$ (right) for the three different projections. $k_{\text {out }}=5 \times 10^{3} \mathrm{~N} / \mathrm{m}$.

\section{uout $(\mu \mathrm{m})$}

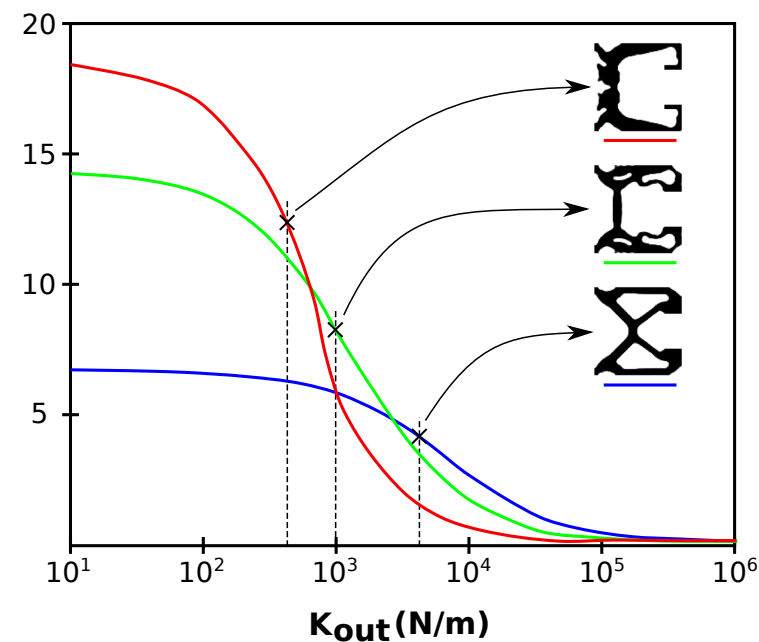

Fig. 6 Evolution of the output displacement with the output stiffness for the blueprint design. 


\section{$\mathbf{F}(\mathbf{N})$}

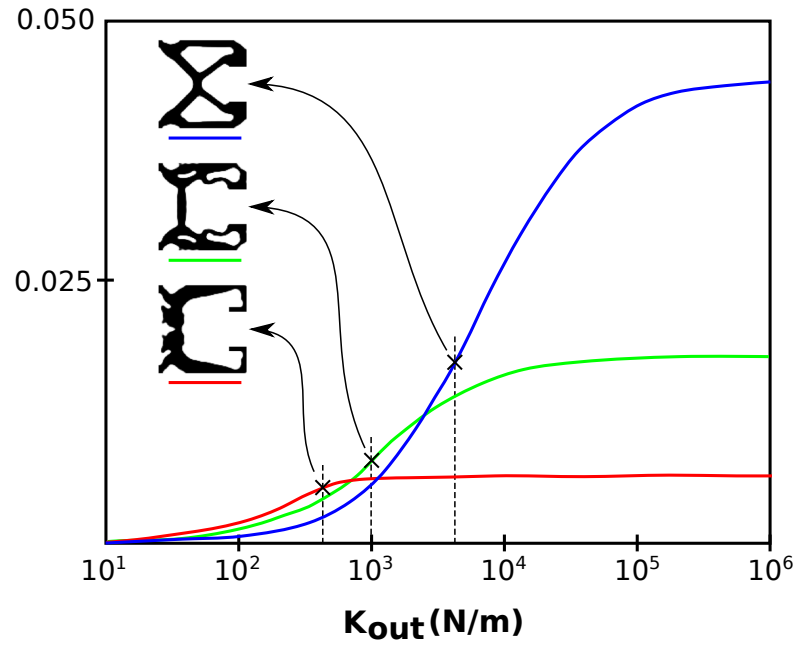

Fig. 7 Evolution of the output force with the output stiffness for the blueprint design.

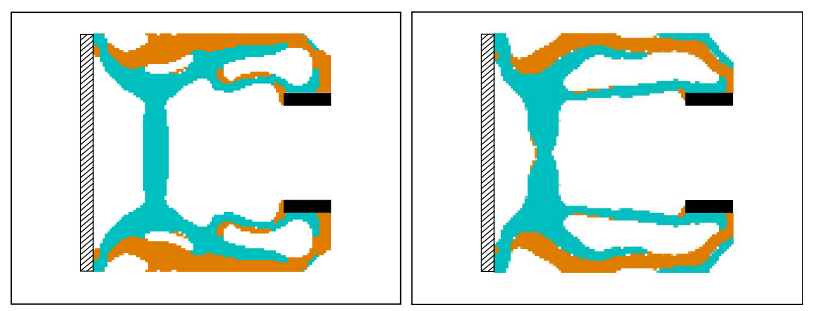

Fig. 8 Optimized microgripper for $k_{\text {out }}=1 \times 10^{3} \mathrm{~N} / \mathrm{m}$ with constraints over the bending at the jaws (left) and without the constraint (right).

mization problem proposed is able to suppress the outof-plane bending produced at the jaws.

Fig. 9 presents the deformation of the grippers commented on above. The device optimized with out-ofplane constraints is shown in Fig. 9 (a). In this example it is easy to check that the out-of-plane deformation is in general small, which is represented with yellow colour. Fig. 9 (b) shows the deformation of the gripper without the constraints. In this case the jaws are blue coloured, which means that this part of the structure is the one with the biggest out-of-plane deformation.

\subsection{Verification with Comsol Multiphysics}

Arguably, the validity of the simplifying linearity assumption for the out-of-plane deformation can be question and the optimization could potentially take adavntage of flaws in the model. Hence, this subsection presents a corroboration of the model using the commercial software Comsol Multiphysics. The gripper is modelled with a fully geometrically non-linear, three-dimensional finite element model using no simplifying assumptions.
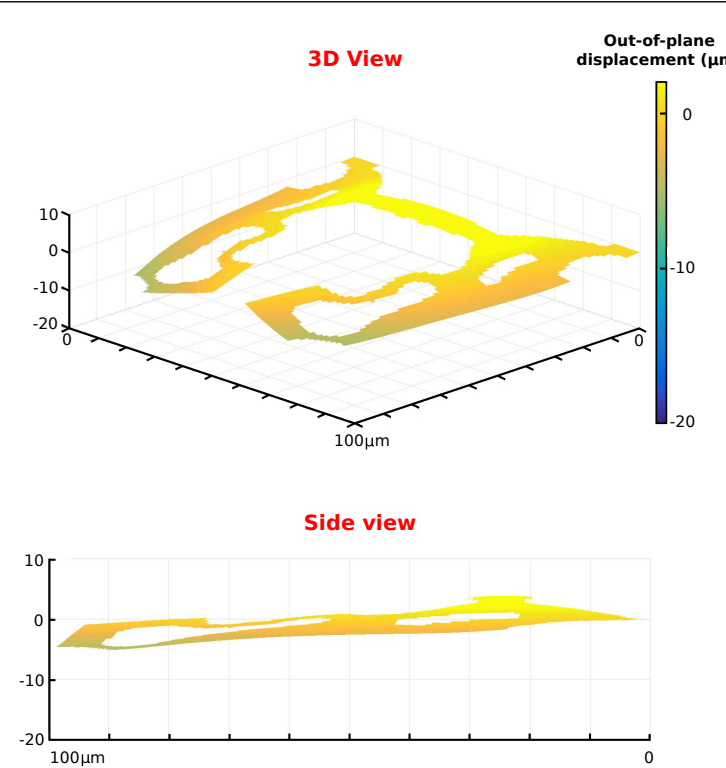

(a) Deformation of the optimized gripper with out-ofplane constraints.
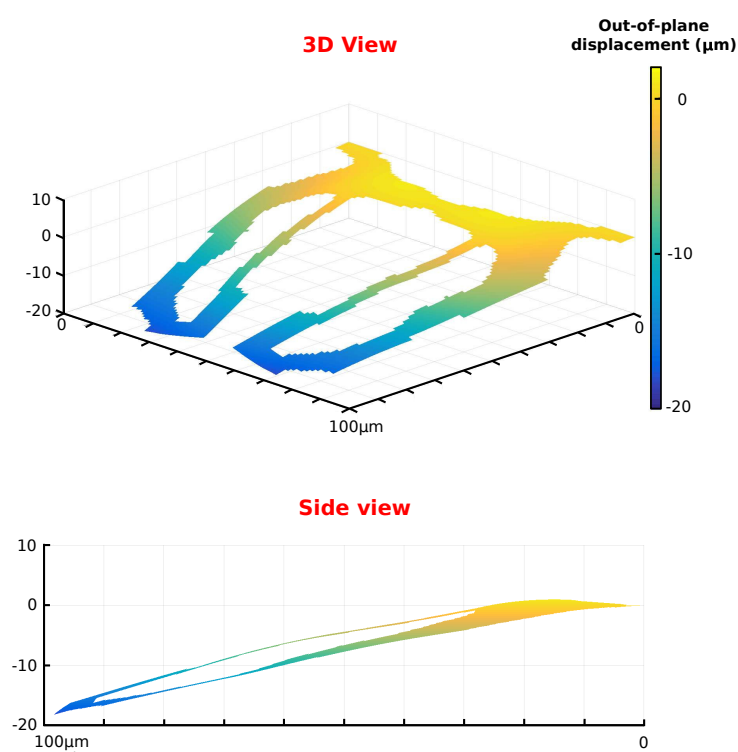

(b) Deformation of the optimized gripper without outof-plane constraints.

Fig. 9 Comparison of deformations between the grippers presented in Fig. 8.

The domain is discretized with 42202 quadratic tetrahedral elements and 247424 degrees of freedom, as shown in Fig. 10. The mesh of the piezoelectric layer is finer due to the smaller thickness.

The deformed gripper is shown in Fig. 11. Resulting displacements are shown in Table 1 which also shows the corresponding displacements obtained from the simplified finite element model used in the optimization. As can be seen from the table, the differences between using full three-dimensional modelling and two-dimensional decoupled plate modelling (with in-plane and out-of- 


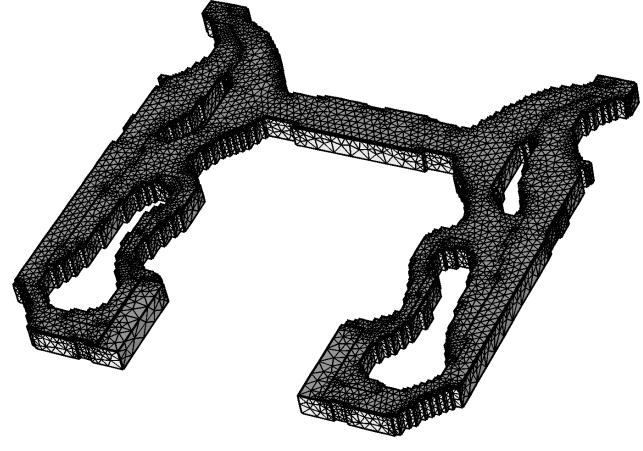

Fig. 10 Meshed 3D realization of the optimized gripper.

\begin{tabular}{|l|c|c|c|}
\cline { 2 - 4 } \multicolumn{1}{c|}{} & $\mathbf{u}_{\mathbf{1}}$ & $\mathbf{u}_{\mathbf{2}}$ & $\mathbf{u}_{\mathbf{3}}$ \\
\hline Comsol & $13.80 \mu \mathrm{m}$ & $0.34 \mu \mathrm{m}$ & $0.96 \mu \mathrm{m}$ \\
\hline Matlab & $13.93 \mu \mathrm{m}$ & $0.44 \mu \mathrm{m}$ & $0.69 \mu \mathrm{m}$ \\
\hline
\end{tabular}

Table 1 Comparison of displacements obtained with Comsol Multiphysics and Matlab for the optimized gripper from Fig. 8 (left).

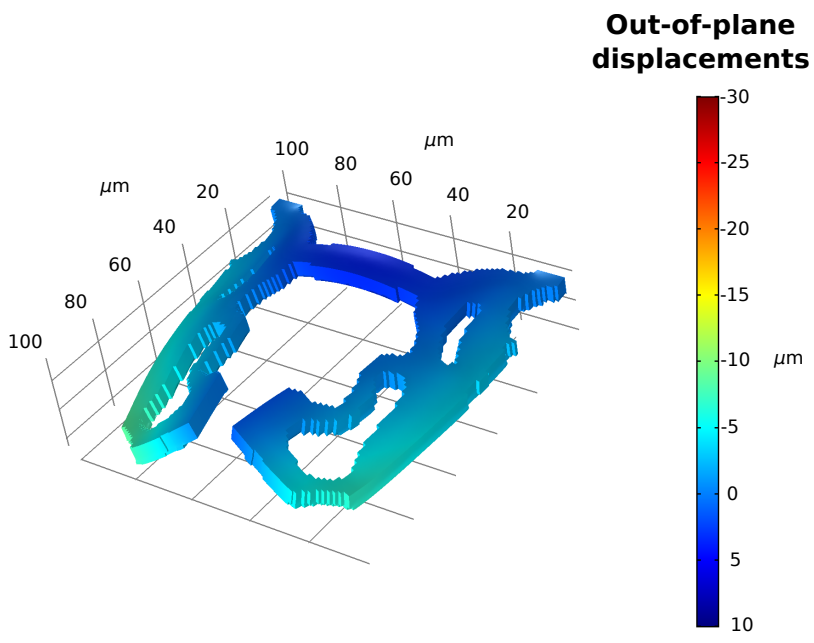

Fig. 11 Deformation of the optimized gripper shown in Fig. 8 (left) modelled using Comsol Multiphysics.

plane motion cases decoupled and linear out-of-plane modelling) are small enough to consider the latter accurate enough.

For comparison, Fig. 12 depicts the deformation of the gripper optimized without out-of-plane deformation constraints from Fig. 8 (right). The displacement values obtained with Comsol Multiphysics and Matlab are shown is Table 2. For this case, remarkably larger discrepancies between the two models are observed. This partly shows that the suggested simplified linear outof-plane modelling is inadequate and partly that inplane and out-of-plane displacements couple when considering large out-of-plane motions. On the other hand,

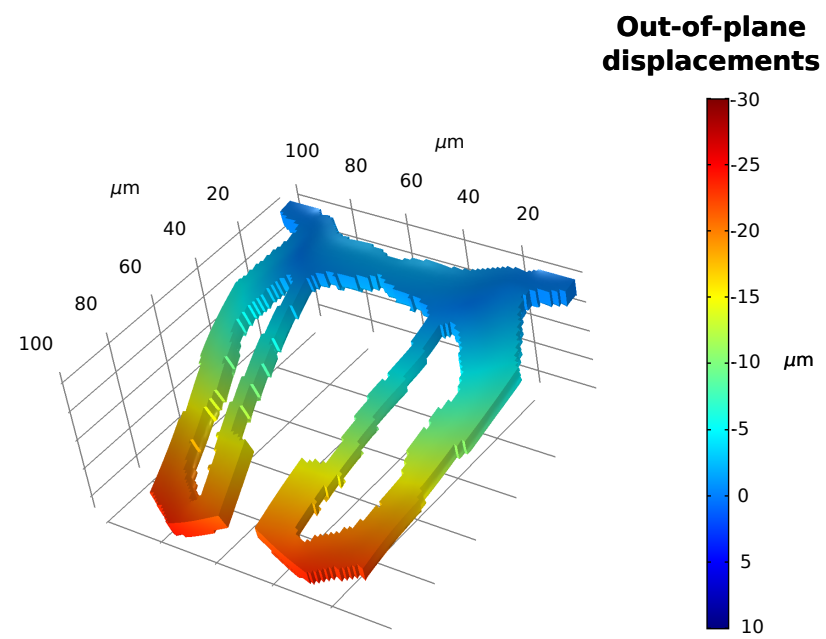

Fig. 12 Deformation of the gripper optimized without outof-plane deformation constraints from Fig. 8 (right) modelled using Comsol Multiphysics.

\begin{tabular}{|c|c|c|c|}
\cline { 2 - 4 } \multicolumn{1}{c|}{} & $\mathbf{u}_{\mathbf{1}}$ & $\mathbf{u}_{\mathbf{2}}$ & $\mathbf{u}_{\mathbf{3}}$ \\
\hline Comsol & $16.10 \mu \mathrm{m}$ & $10.10 \mu \mathrm{m}$ & $15.70 \mu \mathrm{m}$ \\
\hline Matlab & $15.00 \mu \mathrm{m}$ & $8.40 \mu \mathrm{m}$ & $12.80 \mu \mathrm{m}$ \\
\hline
\end{tabular}

Table 2 Comparison of displacements with Comsol Multiphysics and Matlab for the gripper optimized without out-ofplane deformation constraints from Fig. 8 (right).

the two verification examples confirm that for cases with small out-of-plane motions, as dictated by the imposed out-of-plane motion constraints, the simplified and much cheaper and more efficient approach is fully justified.

\section{Conclusions}

In this work piezoelectric microgripper-type actuators are designed by topology optimization. The main novelty introduced is the suppression of out-of-plane bending caused by unsymmetrical lamination of the piezoelectric actuator. This goal is achieved by adding a constraint for each point where the out-of-plane deformation needs to be cancelled. The difficulty arising by only placing one film of piezoelectric material is a real limitation when fabricating at the micro-scale. If the suppression of the out-of-plane deformation is not taken into account, the bending of the gripper jeopardizes functionality. From an optimization point of view it is not possible to suppress the bending in the whole domain. This problem is overcome by suppressing the bending only in four points of interest, placed at the jaws.

The modelling of the in-plane and out-of-plane deformation is different. For the former, the displacements are large compared to the size of the gripper and a 
geometrically non-linear model (we assume large displacements but small strains) is used. For the latter, displacements are small and hence it is not necessary to use a non-linear model and the linear one is used instead to save computational time. An elastic energy interpolation scheme is used to alleviate convergence problems due to excessively distorted low density elements. The validity of the modelling simplifications is confirmed by full three-dimensional and gometrically non-linear modelling of the post-processed design.

A robust formulation provides full length-scale control, avoidance of fragile hinges as well as insensitivity to e.g. under- and over-etching.

Acknowledgements The work of David Ruiz has been founded through grant MTM2013-47053-P from the Spanish Ministerio de Economía y Competitividad. The work of Ole Sigmund has been supported by the Sapere Aude research project 'TOpTEn' (Topology Optimization of Thermal ENergy systems) from the Danish Council for Independent Research, grant: DFF-4005-00320.

\section{References}

Bendsøe and Sigmund, 1999. Bendsøe, M. P. and Sigmund, O. (1999). Material interpolation schemes in topology optimization. Arch Appl Mech, 69(9-10):635-654.

Bendsøe and Sigmund, 2003. Bendsøe, M. P. and Sigmund, O. (2003). Topology optimization: theory, methods and applications. 2nd Edition. Springer.

Bruns and Tortorelli, 2001. Bruns, T. E. and Tortorelli, D. A. (2001). Topology optimization of non-linear elastic structures and compliant mechanisms. Comput Methods Appl Mech Eng, 190(26-27):3443-3459.

Buhl et al., 2000. Buhl, T., Pedersen, C. B. W., and Sigmund, O. (2000). Stiffness design of geometrically nonlinear structures using topology optimization. Struct Multidisc Optim, 19:93 - 104.

Carbonari et al., 2007. Carbonari, R. C., Silva, E. C. N., and Nishiwaki, S. (2007). Optimum placement of piezoelectric material in piezoactuator design. Smart Mater Struct, 16(1):207-220.

Díaz and Kikuchi, 1992. Díaz, A. R. and Kikuchi, N. (1992). Solution to shape and topology eigenvalue optimization problems using a homogenization method. Int $J$ Numer Methods Eng, 35:1487-1502.

Donoso and Sigmund, 2016. Donoso, A. and Sigmund, O. (2016). Topology optimization of piezo modal transducers with null-polarity phases. Struct Multidisc Optim, 53:193203.

Frecker et al., 1997. Frecker, M. I., Ananthasuresh, G. K., Nishiwaki, S., and Kota, S. (1997). Topological synthesis of compliant mechanisms using multi-criteria optimization. Transactions of the ASME, 119(2):238-245.

Gibbs and Fuller, 1992. Gibbs, G. and Fuller, C. (1992). Excitation of thin beams using asymmetric piezoelectric actuators. J Acoust Soc Am, 92(6):3221-3227.

Jensen and Sigmund, 2011. Jensen, J. and Sigmund, O. (2011). Topology optimization for nano-photonics. Laser Photonics Rev, 5(2):308-321.
Jonsmann et al., 1999. Jonsmann, J., Sigmund, O., and Bouwstra, S. (1999). Compliant thermal microactuators. Sens Act A Phys, 76(1-3):463 - 469.

Kang and Tong, 2008a. Kang, Z. and Tong, L. (2008a). Integrated optimization of material layout and control voltage for piezoelectric laminated plates. J Intell Mater Syst Struct, 19:889-904.

Kang and Tong, 2008b. Kang, Z. and Tong, L. (2008b). Topology optimization-based distribution design of actuation voltage in static shape control of plates. Comput Struct, 86(19-20):1885-1893.

Kang et al., 2011. Kang, Z., Wang, R., and Tong, L. (2011). Combined optimization of bi-material structural layout and voltage distribution for in-plane piezoelectric actuation. Comput Methods Appl Mech Eng, 200(13-16):1467-1478.

Kang et al., 2012. Kang, Z., Wang, X., and Luo, Z. (2012). Topology optimization for static shape control of piezoelectric plates with penalization on intermediate actuation voltage. J Mech Des, 134(5):051006.

Kögl and Silva, 2005. Kögl, M. and Silva, E. C. N. (2005). Topology optimization of smart structures: design of piezoelectric plate and shell actuators. Smart Mater Struct, $14(2): 387-399$.

Kucera et al., 2014. Kucera, M., Wistrela, E., Pfusterschmied, G., Ruiz-Díez, V., Manzaneque, T., HernandoGarcía, J., Sánchez-Rojas, J. L., Jachimowicz, A., Schalko, J., Bittner, A., and Schmid, U. (2014). Design-dependent performance of self-actuated and self-sensing piezoelectricaln cantilevers in liquid media oscillating in the fundamental in-plane bending mode. Sens Act B Chem, 200:235244.

Lazarov et al., 2011. Lazarov, B. S., Schevenels, M., and Sigmund, O. (2011). Robust design of large-displacement compliant mechanisms. Mech Sci, 2:175-182.

Luo et al., 2010. Luo, Z., Gao, W., and Song, C. (2010). Design of multi-phase piezoelectric actuators. J Intell Mater Syst Struct, 21(18):1851-1865.

Maute and Frangopol, 2003. Maute, K. and Frangopol, D. M. (2003). Reliability-based design of mems mechanisms by topology optimization. Comput Struct, 81(8-11):813824.

Pedersen et al., 2001. Pedersen, C. B. W., Buhl, T., and Sigmund, O. (2001). Topology synthesis of large-displacement compliant mechanism. Int J Numer Methods Eng, 50:26832705.

Pedersen, 2000. Pedersen, N. L. (2000). Maximization of eigenvalues using topology optimization. Struct Multidisc Optim, 20:2-11.

Qian and Sigmund, 2013. Qian, X. and Sigmund, O. (2013). Topological design of electromechanical actuators with robustness toward over- and under-etching. Comput Methods Appl Mech Eng, 253:237-251.

Ruiz et al., 2016a. Ruiz, D., Bellido, J. C., and Donoso, A. (2016a). Design of piezoelectric modal filters by simultaneously optimizing the structure layout and the electrode profile. Struct Multidisc Optim, 53:715 - 730.

Ruiz et al., 2016b. Ruiz, D., Donoso, A., Bellido, J. C., Kucera, M., Schmid, U., and Sánchez-Rojas, J. L. (2016b) Design of piezoelectric microtransducers based on the topology optimization method. Microsyst Technol, 22(7):17331740.

Sigmund, 1997. Sigmund, O. (1997). On the design of compliant mechanisms using topology optimization. Mech Struct Mach, 25(4):493-524.

Sigmund, 2001a. Sigmund, O. (2001a). Design of multiphysics actuators using topology optimization part i: Onematerial structures. Comput Methods Appl Mech Eng, 190(49-50):6577 - 6604 . 
Sigmund, 2001b. Sigmund, O. (2001b). Design of multiphysics actuators using topology optimization part ii: Twomaterial structures. Comput Methods Appl Mech Eng, 190(49-50):6605 - 6627 .

Sigmund, 2009. Sigmund, O. (2009). Manufacturing tolerant topology optimization. Acta Mechanica Sinica, 25(2):227239.

Sigmund and Jensen, 2003. Sigmund, O. and Jensen, J. S. (2003). Systematic design of phononic band gap materials and structures by topology optimization. Philosophical Transactions of the Royal Society A: Mathematical, Physical and Engineering Sciences, 361:1001-1019.

Sigmund and Torquato, 1997. Sigmund, O. and Torquato, S. (1997). Design of materials with extreme thermal expansion using a three-phase topology optimization method. $J$ Mech Phys Solids, 45(6):1037 - 1067.

Sigmund et al., 1998. Sigmund, O., Torquato, S., and Aksay, I. A. (1998). On the design of 1-3 piezo-composites using topology optimization. J Mater Res, 13(4):1038-1048.

Silva et al., 1997. Silva, E. C. N., Fonseca, J. S. O., and Kikuchi, N. (1997). Optimal design of piezoelectric microstructures. Comput Mech, 19(5):397-410.

Silva and Kikuchi, 1999. Silva, E. C. N. and Kikuchi, N. (1999). Design of piezoelectric transducers using topology optimization. Smart Mater Struct, 8(3):350-364.

Svanberg, 1987. Svanberg, K. (1987). The method of moving asymptotes-a new method for structural optimization. Int $J$ Numer Meth Eng, 24(2):359-373.

Wang et al., 2014. Wang, F., Lazarov, B., Sigmund, O., and Jensen, J. S. (2014). Interpolation scheme for fictitious domain techniques and topology optimization of finite strain elastic problems. Comput Methods Appl Mech Eng, 276:453-472.

Wang et al., 2011. Wang, F., Lazarov, B. S., and Sigmund, O. (2011). On projection methods, convergence and robust formulations in topology optimization. Struct Multidisc Optim, 43(6):767-784.

Zhang et al., 2017. Zhang, W., Chen, J., Zhu, X., Zhou, J., Xue, D., Lei, X., and Guo, X. (2017). Explicit three dimensional topology optimization via moving morphable void (mmv) approach. Comput Methods Apple Mech Engrg, 322:590-614.

Zienkiewicz et al., 2014. Zienkiewicz, O. C., Taylor, R. L., and Fox, D. (2014). The Finite Element Method for Solid and Structural Mechanics. 7th Edition. ButterworthHeinemann. 